\title{
Dynamical $R$-Matrices for the Calogero Models
}

\author{
Michael Forger* ${ }^{* \dagger}$ and Axel Winterhalder ${ }^{\ddagger}$ \\ Departamento de Matemática Aplicada, Instituto de Matemática e Estatística, \\ Universidade de São Paulo, Cx. Postal 66281, BR-05311-970 São Paulo, S.P., Brazil. \\ E-Mail: 'forger@ime.usp.br, winter@ime.usp.bri
}

ABstract: We report on our recent systematic study of the integrability of the Calogero models using the traditional Lax representation due to Moser and, in its general form, to Olshanetsky and Perelomov, based on (semi-) simple Lie algebras or on symmetric pairs, but concentrating on the construction of a dynamical $R$-matrix within this context. Introducing a new family of generators in the Cartan subalgebra of the corresponding simple Lie algebra and assuming the usual functional identities satisfied by the functions that appear in the interaction potential, we show that the conditions guaranteeing integrability can be reduced to a set of algebraic constraints on these generators. As it turns out, these algebraic constraints impose strong restrictions on the (semi-) simple Lie algebra or symmetric pair underlying the construction.

Keywords: 'C̄ălogero models, Lax pairs, Dynamical $\bar{R}$-matrices.

\section{Introduction}

In recent years, the concept of a "dynamical" $R$-matrix - as opposed to that of a "numerical" $R$-matrix which has over the last two decades given rise to an entire new

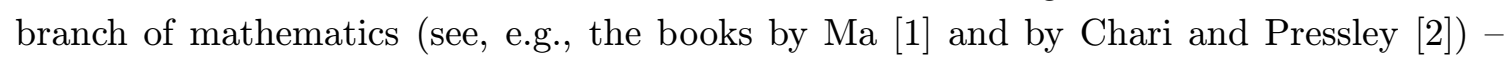
has received increasing attention. Dynamical $R$-matrices first appeared in certain nonultralocal models of $1+1$ - dimensional field theory [3in], in particular the non-linear sigma models 通. Somewhat later, they were also found to arise in mechanics, more specifically

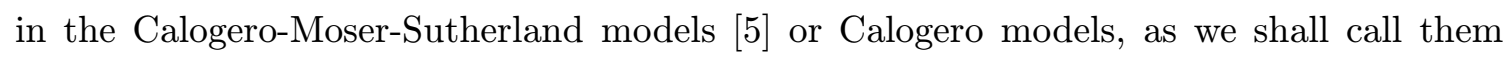
for short [i] For example, it has been repeatedly conjectured that they might be no more than simple "gauge transforms" of standard numerical $R$-matrices. Thus the Calogero models, which

\footnotetext{
${ }^{*}$ Speaker.

${ }^{\dagger}$ Partially supported by CNPq, Brazil

${ }^{\ddagger}$ Work supported by FAPESP, Brazil
} 
are Hamiltonian systems with finitely many degrees of freedom, provide an ideal theoretical laboratory for conceptual investigations concerning the mathematical nature of dynamical $R$-matrices - unlike the non-linear sigma models where such studies are hampered by the jump singularities that plague the Poisson brackets between the entries of the monodromy matrix and require regularization.

In classical mechanics, which is the context of our analysis, the standard tool for handling integrable Hamiltonian systems is the reformulation of their equations of motion as a Lax equation

$$
\dot{L}=[L, M],
$$

where $L$ and $M$ are mappings $L, M: P \rightarrow \mathfrak{g}$ from the phase space $P$ of the model into some Lie algebra $\mathfrak{g}$. Once this has been achieved, it is obvious that the ad-invariant functions on $\mathfrak{g}$ furnish conserved quantities. In the simplest case they are given by the trace polynomials in some irreducible representation,

$$
F_{k}(L)=\operatorname{tr}\left(L^{k}\right)
$$

and it is required that the Hamiltonian of the model be one of them: in the simplest case, it will be the quadratic invariant:

$$
H=\frac{1}{2} \operatorname{tr}\left(L^{2}\right) .
$$

Moreoever, these conserved quantities will be in involution if the entries of $L$ satisfy the so-called fundamental Poisson bracket relation

$$
\left\{L_{1}, L_{2}\right\}=\left[R_{12}, L_{1}\right]-\left[R_{21}, L_{2}\right],
$$

where $R$ is a mapping $R: P \rightarrow U(\mathfrak{g}) \otimes U(\mathfrak{g})$ from the phase space $P$ of the model into the second tensor power of the universal enveloping algebra $U(\mathfrak{g})$ of $\mathfrak{g}$ and we employ the usual tensor notation, where $L_{1}=L \otimes 1, L_{2}=1 \otimes L, R_{12}$ is $R$ and $R_{21}$ is the transpose of $R$ [i] . The fact that $R$ may exhibit non-trivial dependence on the phase space variables is indicated by saying that the $R$-matrix is "dynamical". When $R$ does not depend on the phase space variables, one says that the $R$-matrix is "constant" or "numerical"; an example of this situation is provided by the Toda models.

\section{Calogero models}

The Hamiltonian of the Calogero models describing $n$ particles on a line with pairwise interaction is given by the expression

$$
H(q, p)=\frac{1}{2}\left(\sum_{j=1}^{n} p_{j}^{2}+g^{2} \sum_{\substack{k, l=1 \\ k \neq l}}^{n} w\left(q_{k}-q_{l}\right)^{2}\right),
$$


where $g$ is a coupling constant and $w$ is defined by

$$
w(t)=\left\{\begin{array}{cc}
\frac{1}{t} & \text { for the rational model } \\
\frac{1}{\sin (t)} & \text { for the trigonometric model } \\
\frac{1}{\sinh (t)} & \text { for the hyperbolic model }
\end{array}\right.
$$

For the sake of completeness, we would like to mention that the Calogero models with any one of these three types of potential are collectively referred to as the degenerate Calogero models, as opposed to the elliptic Calogero models where the potential is given by the Weierstrass $\wp$-function.

All these potentials are singular at the origin, so the relative ordering between the particle positions is a constant of motion. Therefore, we may assume without loss of generality that the configuration space of the model is an open subset of $\mathbb{R}^{n}$ defined by some fixed ordering such as, for example,

$$
q_{1}<\ldots<q_{n} .
$$

A Lax pair for this model was found by Moser in the degenerate case and by Krichever in

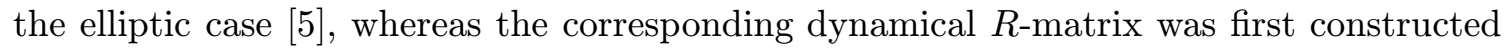

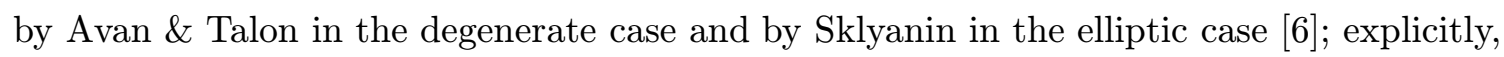
they are in the degenerate case given by

$$
\begin{gathered}
L=\sum_{j=1}^{n} p_{j} E_{j j}+\mathrm{i} g \sum_{\substack{k, l=1 \\
k \neq l}}^{n} w\left(q_{k}-q_{l}\right) E_{k l}, \\
M=\mathrm{ig} \sum_{\substack{k, l=1 \\
k \neq l}}^{n} \frac{w^{\prime \prime}\left(q_{k}-q_{l}\right)}{2 w\left(q_{k}-q_{l}\right)}\left(E_{k k}-\frac{1}{n} 1\right)+\mathrm{i} g \sum_{\substack{k, l=1 \\
k \neq l}}^{n} w^{\prime}\left(q_{k}-q_{l}\right) E_{k l},
\end{gathered}
$$

and

$$
R=-\sum_{\substack{k, l=1 \\ k \neq l}}^{n} w\left(q_{k}-q_{l}\right)\left(E_{k k}-\frac{1}{n} 1\right) \otimes E_{k l}+\sum_{\substack{k, l=1 \\ k \neq l}}^{n} \frac{w^{\prime}\left(q_{k}-q_{l}\right)}{w\left(q_{k}-q_{l}\right)} E_{k l} \otimes E_{l k},
$$

where the symbol $E_{k l}$ denotes the elementary matrix with 1 in the $k^{\text {th }}$ row and $l^{\text {th }}$ column and 0 everywhere else, explicitly given by $\left(E_{k l}\right)_{i j}=\delta_{k i} \delta_{l j}$.

\subsection{Generalization to simple Lie algebras}

Consider a simple complex Lie algebra $\mathfrak{g}$ of rank $r$ with Cartan subalgebra $\mathfrak{h}$ and root system $\Delta$. Let $\mathfrak{g}_{\mathbb{R}}$ be the normal real form of $\mathfrak{g}$ and write $\mathfrak{h}_{\mathbb{R}}$ for the intersection of $\mathfrak{g}_{\mathbb{R}}$ with $\mathfrak{h}$. The algebraic setting encountered above corresponds to the situation where $\mathfrak{g}=\mathfrak{s l}(n, \mathbb{C})$ and $\mathfrak{g}_{\mathbb{R}}=\mathfrak{s l}(n, \mathbb{R})$. In general, the configuration space $Q$ of the model will be (the interior of) a Weyl chamber within $\mathfrak{h}_{\mathbb{R}}$, and the phase space $P$ is its cotangent bundle 
$T^{*} Q=Q \times \mathfrak{h}_{\mathbb{R}}$ which we may identify with its tangent bundle $T Q=Q \times \mathfrak{h}_{\mathbb{R}}$. Choosing an orthonormal basis $\left\{H_{1}, \ldots, H_{r}\right\}$ of $\mathfrak{h}_{\mathbb{R}}$, extended to a Cartan-Weyl basis of $\mathfrak{g}$ with appropriately normalized root generators $E_{\alpha}$, and introducing the vectors $q=\sum_{j=1}^{r} q_{j} H_{j}$ in $Q$ and $p=\sum_{j=1}^{r} p_{j} H_{j}$ in $\mathfrak{h}_{\mathbb{R}}$, we obtain the Hamiltonian

$$
H(q, p)=\frac{1}{2}\left(\sum_{j=1}^{r} p_{j}^{2}+\sum_{\alpha \in \Delta} g_{\alpha}^{2} w(\alpha(q))^{2}\right),
$$

where the coupling constants $g_{\alpha}$ are supposed to be invariant under the Weyl group $W(\mathfrak{g})$. A Lax pair for this more general context was first proposed by Olshanetsky and Pere-

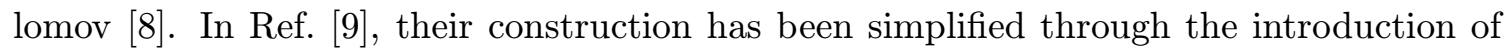
a new set of generators $F_{\alpha}$ in $\mathfrak{h}_{\mathbb{R}}$, over and above the standard $H_{\alpha}$, which allow to write down explicit expressions for the Cartan part of the matrix $M$ as well as for the dynamical $R$-matrix, namely

$$
\begin{gathered}
L=\sum_{j=1}^{r} p_{j} H_{j}+\sum_{\alpha \in \Delta} \mathrm{i} g_{\alpha} w(\alpha(q)) E_{\alpha}, \\
M=-\sum_{\alpha \in \Delta} \mathrm{i} g_{\alpha} \frac{w^{\prime \prime}(\alpha(q))}{2 w(\alpha(q))} F_{\alpha}+\sum_{\alpha \in \Delta} \mathrm{i} g_{\alpha} w^{\prime}(\alpha(q)) E_{\alpha},
\end{gathered}
$$

and

$$
R=\sum_{\alpha \in \Delta} w(\alpha(q)) F_{\alpha} \otimes E_{\alpha}+\sum_{\alpha \in \Delta} \frac{w^{\prime}(\alpha(q))}{w(\alpha(q)} E_{\alpha} \otimes E_{-\alpha} .
$$

As shown in Ref. [in], the required equivalence between the equations of motion derived from the Hamiltonian $(\overline{2} \cdot \overline{7})$ and the Lax equation $\left(\overline{1}_{1}^{1}, \overline{1}_{1}^{\prime}\right)$ as well as the validity of the fundamental Poisson relation (1.) are then guaranteed if and only if one imposes the following set of algebraic constraints on the generators $F_{\alpha}$ :

$$
g_{\alpha} \alpha\left(F_{\beta}\right)-g_{\beta} \beta\left(F_{\alpha}\right)-g_{\alpha+\beta} N_{\alpha, \beta}=0
$$

where the $N_{\alpha, \beta}$ are the structure constants of $\mathfrak{g}$, defined by $\left[E_{\alpha}, E_{\beta}\right]=N_{\alpha, \beta} E_{\alpha+\beta}$. Decomposing $F_{\alpha}$ into its even and odd part $F_{\alpha}^{ \pm}=\frac{1}{2}\left(F_{\alpha} \pm F_{-\alpha}\right)$, these constraints split into two independent sets, namely

$$
\begin{gathered}
g_{\alpha} \alpha\left(F_{\beta}^{+}\right)-g_{\beta} \beta\left(F_{\alpha}^{+}\right)-g_{\alpha+\beta} N_{\alpha, \beta}=0, \\
g_{\alpha} \alpha\left(F_{\beta}^{-}\right)-g_{\beta} \beta\left(F_{\alpha}^{-}\right)=0,
\end{gathered}
$$

the second of which is easily solved by putting $F_{\alpha}^{-}=\lambda g_{\alpha} H_{\alpha}$ where $\lambda$ is an arbitrary constant. The same algebraic constraints are obtained in the case of the elliptic Calogero models.

Explicit calculations show that the only simple complex Lie algebras that admit a solution of these constraints are those of the $A$-series. More precisely, we can state the following 
Theorem 1. For the simple complex Lie algebras $\mathfrak{g}=\mathfrak{s l}(n, \mathbb{C})$ of the A-series, the algebraic constraint (2.12) has the non-trivial solution

$$
F_{i j}^{+}=-\frac{1}{2}\left(E_{i i}+E_{j j}\right)+\frac{1}{n} 1
$$

for $1 \leq i \neq j \leq n$ (where $F_{\alpha_{i j}}$ and $F_{\alpha_{i j}}^{ \pm}$have been abbreviated to $F_{i j}$ and $F_{i j}^{ \pm}$, respectively). In all other cases, the algebraic constraint (2.

The last statement constitutes a no-go theorem that for a long time has been conjectured

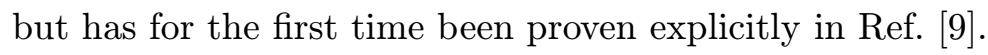

\subsection{Generalization to irreducible symmetric pairs}

Consider an irreducible symmetric pair $(\mathfrak{g}, \theta)$ of $\operatorname{rank} r$, that is, a simple complex Lie algebra $\mathfrak{g}$ together with an involutive automorphism $\theta$, leading to the orthogonal direct decomposition

$$
\mathfrak{g}=\mathfrak{k} \oplus \mathfrak{m}
$$

of $\mathfrak{g}$ into the $\theta=+1$ - eigenspace $\mathfrak{k}$ which is a subalgebra and the $\theta=-1$ - eigenspace $\mathfrak{m}$, with the standard commutation relations $[\mathfrak{k}, \mathfrak{k}] \subset \mathfrak{k},[\mathfrak{k}, \mathfrak{m}] \subset \mathfrak{m}$ and $[\mathfrak{m}, \mathfrak{m}] \subset \mathfrak{k}$. We choose a maximally noncompact $\theta$-invariant Cartan subalgebra $\mathfrak{h}$ of $\mathfrak{g}$, leading to an analogous orthogonal direct decomposition

$$
\mathfrak{h}=\mathfrak{b} \oplus \mathfrak{a}
$$

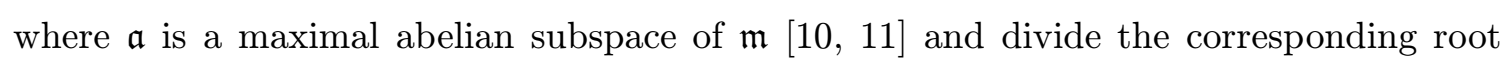
system $\Delta$ into two subsystems - the system $\Delta_{0}$ of roots that vanish on $\mathfrak{a}$ and the system $\tilde{\Delta}$ of all other roots. Let $\mathfrak{g}_{0}$ be the unique real form of $\mathfrak{g}$ such that the restriction of $\theta$ to $\mathfrak{g}_{0}$ is the Cartan involution of $\mathfrak{g}_{0}$, and write $\mathfrak{k}_{0}, \mathfrak{m}_{0}, \mathfrak{h}_{0}, \mathfrak{b}_{0}$ and $\mathfrak{a}_{0}$ for the intersection of $\mathfrak{g}_{0}$ with $\mathfrak{k}, \mathfrak{m}, \mathfrak{h}, \mathfrak{b}$ and $\mathfrak{a}$, respectively; then $\mathfrak{h}_{0}=\mathfrak{b}_{0} \oplus \mathfrak{a}_{0}$ whereas $\mathfrak{h}_{\mathbb{R}}=\mathfrak{i} \mathfrak{b}_{0} \oplus \mathfrak{a}_{0}$. The configuration space $Q$ of the model is now (the interior of) a Weyl chamber in $\mathfrak{a}_{0}$ and hence the phase space $P$ is its cotangent bundle $T^{*} Q=Q \times \mathfrak{a}_{0}^{*}$ which we may identify with its tangent bundle $T Q=Q \times \mathfrak{a}_{0}$. Choosing an orthonormal basis $\left\{H_{1}, \ldots, H_{r}\right\}$ of $\mathfrak{a}_{0}$, complemented by an orthonormal basis $\left\{H_{r+1}, \ldots, H_{r+s}\right\}$ of $\mathfrak{i b}_{0}$ and extended to a Cartan-Weyl basis of $\mathfrak{g}$ with appropriately normalized root generators $E_{\alpha}$ satisfying the condition ${ }^{1} \theta E_{\alpha}=E_{\theta \alpha}$, and introducing the vectors $q=\sum_{j=1}^{r} q_{j} H_{j}$ in $Q$ and $p=\sum_{j=1}^{r} p_{j} H_{j}$ in $\mathfrak{a}_{0}$ as before, we obtain the Hamiltonian

$$
H(q, p)=\frac{1}{2}\left(\sum_{j=1}^{r} p_{j}^{2}+\sum_{\alpha \in \tilde{\Delta}} g_{\alpha}^{2} w(\alpha(q))^{2}\right),
$$

which is formally the same as before, provided we assume the coupling constants $g_{\alpha}$ to vanish for $\alpha \in \Delta_{0}$; moreover, we suppose them to be invariant under the automorphism $\theta$ $\left(g_{\theta \alpha}=g_{\alpha}\right)$ as well as under the Weyl group $W(\mathfrak{g}, \theta)$ associated with the symmetric pair

\footnotetext{
${ }^{1}$ In the Appendix of Ref. [ $\left[\bar{g}_{1}^{1}\right]$, it is (erroneously) claimed that the root generators $E_{\alpha}$ can always be chosen to satisfy this condition, while it is in general only possible to achieve this up to signs. See the erratum to

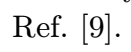


$(\mathfrak{g}, \theta)$, which is defined to be the centralizer of $\theta$ in the full Weyl group $W(\mathfrak{g})$ of $\mathfrak{g}$. Again, a Lax pair for this case was first proposed by Olshanetsky and Perelomov [i $[\overline{-1}]$, and again, their construction has been simplified in Ref. [9-9] through the introduction of a new set of generators in $\mathrm{ib}_{0}$, denoted here by $K_{\alpha}$ rather than $F_{\alpha}$, invariant under the automorphism $\theta$ $\left(K_{\theta \alpha}=K_{\alpha}\right)$, which allow to write down explicit expressions for the Cartan part of the matrix $M$ as well as for the dynamical $R$-matrix, namely

$$
\begin{gathered}
L=\sum_{j=1}^{r} p_{j} H_{j}+\sum_{\alpha \in \tilde{\Delta}} \mathrm{i} g_{\alpha} w(\alpha(q)) E_{\alpha}, \\
M=-\sum_{\alpha \in \tilde{\Delta}} \mathrm{i} g_{\alpha} \frac{w^{\prime \prime}(\alpha(q))}{2 w(\alpha(q))} K_{\alpha}+\sum_{\alpha \in \tilde{\Delta}} \mathrm{i} g_{\alpha} w^{\prime}(\alpha(q)) E_{\alpha},
\end{gathered}
$$

and

$$
R=\sum_{\alpha \in \tilde{\Delta}} w(\alpha(q)) K_{\alpha} \otimes E_{\alpha}+\frac{1}{2} \sum_{\alpha \in \tilde{\Delta}} \frac{w^{\prime}(\alpha(q))}{w(\alpha(q)}\left(E_{\theta \alpha} \otimes E_{-\alpha}+E_{\alpha} \otimes E_{-\alpha}\right) .
$$

As before, the required equivalence between the equations of motion derived from the Hamiltonian (2.17i) and the Lax equation (1, $\left.11_{1}^{\prime}\right)$ as well as the validity of the fundamental Poisson relation $(\overline{1}-\overline{4} \mathbf{4})$ can be shown $\left[\begin{array}{l}\overline{9} \\ 1\end{array}\right]$ to hold if and only if one imposes the following set of algebraic constraints on the generators $K_{\alpha}$ :

$$
g_{\alpha} \alpha\left(K_{\beta}\right)-g_{\beta} \beta\left(K_{\alpha}\right)-\frac{1}{2}\left(g_{\alpha+\beta} N_{\alpha, \beta}+g_{\theta \alpha+\beta} N_{\theta \alpha, \beta}\right)=0,
$$

where the $N_{\alpha, \beta}$ are as before but are required to be $\theta$-invariant $\left(N_{\theta \alpha, \theta \beta}=N_{\alpha, \beta}\right)$. Again, decomposing $K_{\alpha}$ into its even and odd part $K_{\alpha}^{ \pm}=\frac{1}{2}\left(K_{\alpha} \pm K_{-\alpha}\right)$, these constraints split into two independent sets, namely

$$
\begin{gathered}
g_{\alpha} \alpha\left(K_{\beta}^{+}\right)-g_{\beta} \beta\left(K_{\alpha}^{+}\right)-\frac{1}{2}\left(g_{\alpha+\beta} N_{\alpha, \beta}+g_{\theta \alpha+\beta} N_{\theta \alpha, \beta}\right)=0, \\
g_{\alpha} \alpha\left(K_{\beta}^{-}\right)-g_{\beta} \beta\left(K_{\alpha}^{-}\right)=0,
\end{gathered}
$$

the second of which is easily solved by putting $K_{\alpha}^{-}=\lambda g_{\alpha}\left(H_{\alpha}\right)_{\mathfrak{b}}=\frac{1}{2} \lambda g_{\alpha}\left(H_{\alpha}+\theta H_{\alpha}\right)$ where $\lambda$ is an arbitrary constant. The same algebraic constraints are obtained in the case of the elliptic Calogero models.

The problem of solving these constraints or else of proving that there is no solution has not yet been tackled in full generality. Complete results are however available for the symmetric pairs of the $A I I I$-series:

Theorem 2. For the symmetric pairs of the AIII-series corresponding to the complex Grassmannians, that is, the symmetric spaces $S U(p+q) / S(U(p) \times U(q))$ (compact type) or $S U(p, q) / S(U(p) \times U(q))$ (noncompact type), the algebraic constraint (20.2li) admits a solution if and only if

$$
|q-p| \leqslant 1
$$

which in terms of the explicit matrix representation employed in Ref. $[\bar{g}]$ is given as follows. Assuming that $p \leqslant q$, let $n=p+q$ and, for $1 \leq i \leq n$, define $\theta(i)$ by $\theta(i)=i+q$ for $1 \leqslant i \leqslant p, \theta(i)=i$ for $p+1 \leqslant i \leqslant q$ (applicable only when $p<q$ ) and $\theta(i)=i-q$ for $q+1 \leqslant i \leqslant n$. Then abbreviating $K_{\alpha_{i j}}$ and $K_{\alpha_{i j}}^{ \pm}$to to $K_{i j}$ and $K_{i j}^{ \pm}$, respectively, we have 
- if $q=p, n=2 p$, then

$$
K_{i j}^{+}=-\frac{1}{4}\left(E_{i i}+E_{j j}+E_{\theta(i) \theta(i)}+E_{\theta(j) \theta(j)}\right)+\frac{1}{n} 1
$$

for $1 \leq i \neq j \leq n$, independently of the values of the two free coupling constants $g$ and $g_{2}$ allowed by Weyl group invariance;

- if $q=p+1, n=2 p+1$, then

$$
K_{i j}^{+}=-\frac{1}{4}\left(E_{i i}+E_{j j}+E_{\theta(i) \theta(i)}+E_{\theta(j) \theta(j)}\right)+\frac{1}{n} 1
$$

for $1 \leq i \neq j \leq n$ such that $i, j \neq p+1$, while

$$
K_{i, p+1}^{+}=-\frac{g_{1}}{4 g}\left(E_{i i}+E_{\theta(i) \theta(i)}\right)-\frac{g}{2 g_{1}} E_{p+1, p+1}+\frac{1}{n}\left(\frac{g_{1}}{2 g}+\frac{g}{2 g_{1}}\right) 1
$$

for $1 \leq i \leq n$ such that $i \neq p+1$, provided the three free coupling constants $g, g_{1}$ and $g_{2}$ allowed by Weyl group invariance satisfy the relations $g \neq 0, g_{1} \neq 0$ and

$$
g_{1}^{2}-2 g^{2}+g g_{2}=0 .
$$

This result allows to construct a Lax representation with a dynamical $R$-matrix for the Calogero models, with appropriately chosen coupling constants, based on any of the classical root systems not covered by Theorem 1: $B_{n}, C_{n}, D_{n}$ and $B C_{n}$. For more details, see Ref. [9יר].

\section{Dynamical versus numerical $R$-matrices}

Within the general context presented in the previous section, we have recently investigated whether and to what extent the dynamical $R$-matrices encountered in the Calogero models can, by means of appropriate gauge transformations, be reduced to numerical $R$-matrices, whose mathematical interpretation in terms of Lie bialgebras is well known [2] $]$. This analysis has first been carried out for the original Calogero models based on the $A$-series of simple Lie algebras, initially by Hou and Yang in the elliptic case [i] a somewhat different method, by Fehér and Pusztai in the degenerate case [1] follows, we shall briefly summarize our main results, which extend those of Fehér and Pusztai; details can be found in Ref. [1] 1 ind

\subsection{Gauge transformations}

Given a Lax pair and an $R$-matrix as in Sect. 1 , we are free to submit them to a gauge transformation by an arbitrary function $g: P \rightarrow G$ on the phase space $P$ of the model with values in some connected Lie group $G$ with Lie algebra $\mathfrak{g}$, under which the Lax matrix and the $R$-matrix transform according to $L \rightarrow L^{\prime}$ and $R \rightarrow R^{\prime}$, with

$$
L^{\prime}=g L g^{-1}
$$




$$
R_{12}^{\prime}=g_{1} g_{2}\left(R_{12}+g_{1}^{-1}\left\{g_{1}, L_{2}\right\}+\frac{1}{2}\left[g_{1}^{-1} g_{2}^{-1}\left\{g_{1}, g_{2}\right\}, L_{2}\right]\right) g_{1}^{-1} g_{2}^{-1},
$$

where the transformation law for $R$ is determined by the requirement that the fundamental Poisson bracket relation (1.4) should be preserved. In the case of the Calogero models where the $R$-matrix depends only on the position but not on the momentum variables, it is natural to make the same assumption for the function $g$; then using that the Lax matrix is an affine function of the momentum variables of the form

$$
L=\sum_{j=1}^{r} p_{j} H_{j}+\sum_{\alpha \in \Delta} L_{\alpha} E_{\alpha}
$$

where the functions $L_{\alpha}$ depend only on the position but not on the momentum variables, the transformation law for the $R$-matrix simplifies to

$$
R^{\prime}=(g \otimes g)\left(R-\sum_{j=1}^{r} A_{j} \otimes H_{j}\right)\left(g^{-1} \otimes g^{-1}\right),
$$

where $A_{j}=g^{-1} \partial_{j} g$, so that

$$
\partial_{k} A_{l}-\partial_{l} A_{k}+\left[A_{k}, A_{l}\right]=0 .
$$

For $R^{\prime}$ to be numerical, all partial derivatives $\partial_{k} R^{\prime}$ have to vanish and we obtain

$$
\partial_{k} R-\sum_{j=1}^{r} \partial_{j} A_{k} \otimes H_{j}-\left[R, A_{k} \otimes 1+1 \otimes A_{k}\right]+\sum_{j=1}^{r} A_{j} \otimes\left[H_{j}, A_{k}\right]=0 .
$$

The last two equations are further evaluated by decomposing the gauge potentials $A_{j}$ into their Cartan part and root part:

$$
A_{j}=A_{j}^{\mathfrak{h}}+\sum_{\alpha \in \Delta} A_{j}^{\alpha} E_{\alpha} .
$$

As it turns out, the standard functional equations satisfied by the function $w$ in eq. (2.2)

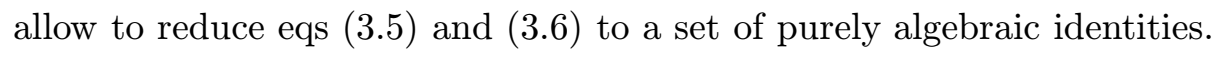

\subsection{Results for simple Lie algebras}

In the case of simple complex Lie algebras, there are two important algebraic identities that must be satisfied by the generators $F_{\alpha}$ appearing in the expression (2.10i) for the $R$-matrix: one states that (except for a normalization and/or sign factor), its odd part $F_{\alpha}^{-}$equals the standard Cartan generator $H_{\alpha}$ while its even part $F_{\alpha}^{+}$is orthogonal to $H_{\alpha}$, whereas the second can be written as a vector constraint in $\mathfrak{h}_{\mathbb{R}}$ :

$$
\begin{gathered}
\alpha\left(F_{\beta}\right) F_{\alpha}-\beta\left(F_{\alpha}\right) F_{\beta}=N_{\alpha, \beta} F_{\alpha+\beta} \\
\text { for } \alpha, \beta \in \Delta \text { such that } \beta \neq \pm \alpha .
\end{gathered}
$$

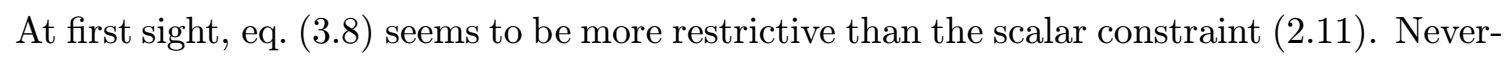
theless, detailed calculations show that it is not: both constraints have the same solutions, forcing $\mathfrak{g}$ to belong to the $A$-series. Summarizing, we can state the following 
Theorem 3. The degenerate Calogero model associated with a simple complex Lie algebra $\mathfrak{g}$ admits a gauge transformation $g$ from the standard Lax pair of Olshanetsky and Perelomov and the dynamical $R$ matrix given by eq. (2.1d) to a new Lax pair with a numerical $R$ matrix if and only if $\mathfrak{g}$ belongs to the $A$-series. In this case, the solutions of eqs (2.1 and (3.8) coincide, and the gauge transformation potential takes the form (3. (3) with

$$
A_{k}^{\alpha}(q)=w(\alpha(q))\left(H_{k}, F_{\alpha}\right)
$$

and

$$
A_{k}^{\mathfrak{h}}(q)=\sum_{\alpha \in \Delta} \frac{w^{\prime}(\alpha(q))}{w(\alpha(q))}\left(H_{k}, F_{-\alpha}\right) F_{\alpha}
$$

The same statement holds for the elliptic Calogero models, despite the fact that in this case, eqs $(\overline{3} \cdot \overline{3})$ and $\left(\overline{3}-\bar{b}_{i}\right)$ lead to additional algebraic constraints which however turn out not to impose any further restrictions.

\subsection{Results for irreducible symmetric pairs}

In the case of irreducible symmetric pairs, the situation is somewhat more complicated. First of all, we must introduce two sets of generators in the Cartan subalgebra: one is the set of generators $K_{\alpha}$ belonging to $\mathrm{ib}_{0}$ that appear in the formula (2.20) for the $R$-matrix and the other is a set of generators $M_{\alpha}$ belonging to $\mathfrak{a}_{0}$ that appear in the explicit form of the gauge potential $(\overline{3})$ to be given below. There are then two important algebraic identities that must be satisfied by the generators $K_{\alpha}$ and $M_{\alpha}$ : one states that (except for a normalization and/or sign factor), their odd parts $K_{\alpha}^{-}$and $M_{\alpha}^{-}$equal the projection of the standard Cartan generator $H_{\alpha}$ onto $\mathfrak{i b}_{0}$ and onto $\mathfrak{a}_{0}$, respectively, while their even parts $K_{\alpha}^{+}$and $M_{\alpha}^{+}$are orthogonal to $H_{\alpha}$, whereas the second can be written as two vector constraints, one in $\mathrm{ib}_{0}$ and and one in $\mathfrak{a}_{0}$ :

$$
\begin{gathered}
\alpha\left(K_{\beta}\right) K_{\alpha}-\beta\left(K_{\alpha}\right) K_{\beta}=\frac{1}{2}\left(N_{\alpha, \beta} K_{\alpha+\beta}+N_{\theta \alpha, \beta} K_{\theta \alpha+\beta}\right) \\
\alpha\left(K_{\beta}\right) M_{\alpha}-\beta\left(M_{\alpha}\right) M_{\beta}=\frac{1}{2}\left(N_{\alpha, \beta} M_{\alpha+\beta}-N_{\theta \alpha, \beta} M_{\theta \alpha+\beta}\right) \\
\text { for } \alpha, \beta \in \Delta \text { such that } \beta \neq \pm \alpha, \beta \neq \pm \theta \alpha .
\end{gathered}
$$

Moreover, it turns out that the possibility to solve the pertinent set of algebraic identities depends on two additional constraints on the structure of the underlying root system.

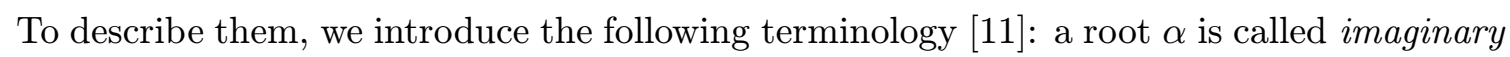
if $\theta \alpha=\alpha$, real if $\theta \alpha=-\alpha$ and complex if $\theta \alpha$ and $\alpha$ are linearly independent. Two roots $\alpha$ and $\beta$ are said to be strongly orthogonal if neither $\alpha+\beta$ nor $\alpha-\beta$ is a root. Then the additional constraints are

- there are no imaginary roots, i.e., $\Delta_{0}=\emptyset$ and $\tilde{\Delta}=\Delta$,

- for every complex root $\alpha$ in $\Delta, \theta \alpha$ and $\alpha$ are strongly orthogonal, i.e., $\theta \alpha \pm \alpha$ does not belong to $\Delta$. 
Setting

$$
F_{\alpha}=K_{\alpha}+M_{\alpha},
$$

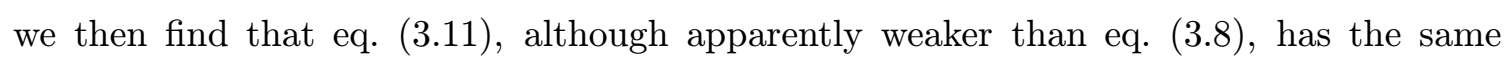
solutions, forcing $\mathfrak{g}$ to belong to the $A$-series. Moreover, the first of the two additional constraints above (together with the requirement that it should be possible to choose the root generators $E_{\alpha}$ so as to satisfy $\theta E_{\alpha}=E_{\theta \alpha}$, which is a necessary condition for integrability) exclude the symmetric pairs of the $A I$-series, of the $A I I$-series and of the $A I I I$-series when $|p-q|>1$. Finally, the second of the two additional constraints above excludes the symmetric pairs of the $A I I I$-series when $|p-q|=1$. Summarizing, we can state the following

Theorem 4. The degenerate Calogero model associated with an irreducible symmetric pair $(\mathfrak{g}, \theta)$ admits a gauge transformation $g$ from the standard Lax pair of Olshanetsky and Perelomov and the dynamical $R$-matrix given by eq. (2.20 to a new Lax pair with a numerical $R$-matrix if and only if $(\mathfrak{g}, \theta)$ corresponds to the complex Grassmannian $S U(2 n) / S(U(n) \times U(n)$ (compact type) or $S U(n, n) / S(U(n) \times U(n))$ (noncompact type). In this case, the gauge transformation potential takes the form (1,

$$
A_{k}^{\alpha}(q)=w(\alpha(q))\left(H_{k}, M_{\alpha}\right)
$$

and

$$
A_{k}^{\mathfrak{h}}(q)=\sum_{\alpha \in \Delta} \frac{w^{\prime}(\alpha(q))}{w(\alpha(q))}\left(H_{k}, M_{-\alpha}\right) K_{\alpha} .
$$

The same statement holds for the elliptic Calogero models, despite the fact that in this

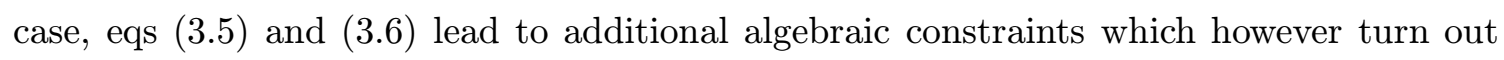
not to impose any further restrictions.

\section{Conclusions and outlook}

The main conclusions to be drawn from the results described above are the following.

- In Ref. [ind the question of integrability of the Calogero models associated with irreducible symmetric pairs has been definitely settled for the $A I I I$-series, corresponding to the complex Grassmannians $S U(p, q) / S(U(p) \times U(q))$ : as shown there, integrability holds if and only if $|p-q| \leqslant 1$. This is sufficient to cover all classical root systems that cannot be handled in a direct Lie algebraic approach, including the non-reduced $B C_{n^{-}}$system as well as the reduced $B_{n^{-}}, C_{n^{-}}$and $D_{n^{-}}$systems, provided the coupling constants are appropriately chosen. A classification of integrability for the remaining irreducible symmetric pairs (see Table V in [i] ${ }_{1}^{1} \overline{0}_{1}^{\prime \prime}$ p. 518]) is still missing. However, we strongly suspect that the $A I I I$ - series in fact provide the only case which allows for a non-trivial solution.

- In Ref. [1] $1 \overline{4}$, it is shown that requiring the dynamical $R$-matrix for an integrable Calogero model associated with some irreducible symmetric pair to be the gauge 
transform of a numerical $R$-matrix imposes additional restrictions on the underlying root system. These additional restrictions are strong enough to rule out all Calogero models except those based on the complex Grassmannians $S U(p, q) / S(U(p) \times U(q))$ of the $A I I I$ - series and, more importantly, also rule out the case $|p-q|=1$, leaving only the case $p=q$ : this includes the models of type $C_{n}$ and $D_{n}$ but excludes those of type $B_{n}$ and $B C_{n}$.

- The results of Ref. [1] $1 \overline{4}$ definitely confirm the position that we adopted at the very begining of our investigation, namely that the concept of a dynamical $R$-matrix cannot be entirely reduced to the more traditional one of a numerical $R$-matrix that has only been concealed by "twisting" it with some gauge transformation. Moreover, it should be noted that even when this is the case, this gauge transformation may itself assume a dynamical role of its own - just like the transformation to actionangle variables in an integrable Hamiltonian system (in the sense of Liouville), which absorbs much of its dynamics.

In view of the picture that emerges from this analysis, various problems of mathematical nature gain renewed importance. Among these are questions such as the following.

- What are the algebro-differential constraints to be satisfied by a dynamical $R$-matrix? These constraints are usually referred to as the "dynamical Yang-Baxter equation", which extends and generalizes the usual Yang-Baxter equation for numerical $R$ matrices. However, it seems to us that the precise form of this equation and its appropriate mathematical interpretation are not entirely clear. In the context of the Calogero models studied here, a natural candidate is the following:

$$
\left\{L_{2}, R_{13}\right\}-\left\{L_{3}, R_{12}\right\}+\left[R_{12}, R_{23}\right]+\left[R_{12}, R_{13}\right]+\left[R_{32}, R_{13}\right]=0 .
$$

It has the advantage of being invariant under gauge transformations [i]1$\overline{1} \overline{1}]$, but it does not seem to coincide with the equation proposed by other authors [i] $\left.\overline{6}_{1}^{6}\right]$. At the present stage of investigation, we do not know what are the relations between the various kinds of dynamical Yang-Baxter equations.

- What is the origin of the constraints that restrict integrability of Calogero models?

We hope to find an answer to the problem of characterizing the mathematical structure behind the dynamical $R$-matrices of the Calogero models by studying their relation with the geodesic flow on certain symmetric spaces and also with the recently introduced spin Calogero models, a relation that is a typical application of the

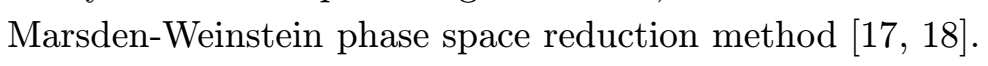

\section{References}

[1] Zhong-Qi Ma: Yang-Baxter Equation and Quantum Enveloping Algebras, World Scientific, Singapore 1993.

[2] V. Chari \& A. Pressley: A Guide to Quantum Groups, Cambridge University Press, Cambridge 1994. 
[3] L.D. Faddeev \& L.A. Takhtajan, Hamiltonian Methods in the Theory of Solitons, Springer-Verlag, Berlin, 1987.

[4] H.J. de Vega, H. Eichenherr \& J.M. Maillet: Classical and Quantum Algebras of Non-Local Charges in Sigma Models, 'Comm. Math. Phys. 92 1984$) 507524$;

J.-M. Maillet: Kac-Moody Algebra and Extended Yang-Baxter Relations in the

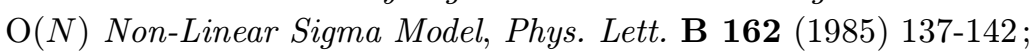

J.-M. Maillet: Hamiltonian Structures for Integrable Classical Theories from

Graded Kac-Moody Algebras, 'P

J.-M. Maillet: New Integrable Canonical Structures in Two-Dimensional Models, Nucl.Phys. B 269-(1986) $54 \mathbf{4} 76$;

M. Bordemann, M. Forger, J. Laartz \& U. Schäper: The Lie-Poisson Structure of Integrable

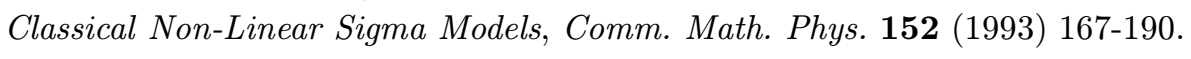

[5] F. Calogero: Solution of the One-Dimensional n-Body Problem with Quadratic

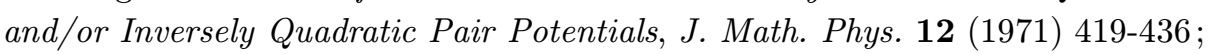

B. Sutherland: Exact Results for a Quantum Many-Body Problem in One Dimension,

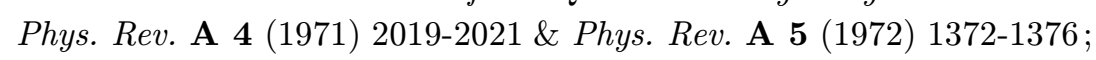

J. Moser: Three Integrable Hamiltonian Systems Connected with Isospectral Deformations, Ádv. Math $\mathbf{1} \mathbf{6}(\overline{1} \overline{9} \overline{5}) \overline{1}-23$;

I.M. Krichever: Elliptic Solutions of the Kadomtsev-Petviashvili Equation and Integrable Systems of Particles, 'Funct. Anal. Appl. 14_(1980) 282-290.

[6] J. Avan \& M. Talon: Classical R-Matrix Structure for the Calogero Model, Phys. Lett. B $\mathbf{3 0 3}(199 \overline{3}) \overline{3} 3-37$

E.K. Sklyanin: Dynamical R-Matrices for the Elliptic Calogero-Moser Model, Alg. Anal. 6 (1994) 227, hep-th/9308060.

[7] O. Babelon \& C.-M. Viallet: Hamiltonian Structures and Lax Equations,

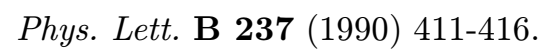

[8] M.A. Olshanetsky \& A.M. Perelomov: Completely Integrable Hamiltonian Systems Connected with Semisimple Lie Algebras, Invent. Math. $\mathbf{3} 7$ (1976) $9 \overline{3}-108$;

M.A. Olshanetsky \& A.M. Perelomov: Classical Integrable Finite-Dimensional Systems Related to Lie Algebras, Phys. Rept. 71 (1981) 313 - 400 ;

A.M. Perelomov: Integrable Systems of Classical Mechanics and Lie Algebras, Vol. 1, Birkhäuser, Basel, 1990.

[9] M. Forger \& A. Winterhalder: Dynamical R-Matrices for Calogero Models, iNucl. Phys. B 621

[10] S. Helgason: Differential Geometry, Lie Groups and Symmetric Spaces, Academic Press, New York 1978.

[11] A. Knapp: Lie Groups Beyond an Introduction, Birkhäuser, Basel 1996.

[12] B.Y. Hou \& W.L. Yang: The Non-Dynamical R-Matrix Structure of the Elliptic Calogero-Moser Model, LLett. Math. Phys. 44 (1998) 35r-41, solv-int/9711008;

The Non-Dynamical R-Matrix Structure of the Elliptic $A_{n-1}$ Calogero-Moser Model, 'L-' P'hys.'

: - .

[13] L. Fehér and B.G. Pusztai: On the Classical R-Matrix of the Degenerate Calogero-Moser Models, Czech J. Phys. 50 (2000) 59-64, math-ph/9912021v2; The Non-Dynamical R-Matrices of the Degenerate Calogero-Moser Models, iJ. Phys. A 3 3 $(2000) 7739$ r 7759 , math-ph/0005021v2. 
[14] M. Forger \& A. Winterhalder: From Dynamical to Numerical R-matrices: A Case Study for the Calogero Models, Preprint IME-USP RT-MAP-0202, December 2002, hep-th/0212273.

[15] M. Forger \& A. Winterhalder: Gauge Transformations of the Dynamical Yang-Baxter Equation, in preparation.

[16] P. Etingof \& A. Varchenko: Geometry and Classification of the Solutions of the Classical Dynamical Yang-Baxter Equation, 'Comm. Math. Phys. 192 (1998) 77tr120.

[17] J. Avan, O. Babelon \& M. Talon: Construction of the Classical R-Matrices for the Toda and Calogero Models, Alg. Anal. 6 (1994) 67, hep-th/9306102:

[18] Luen-Chau Li \& Ping Xu: Spin Calogero-Moser Systems Associated with Simple Lie Algebras, C. R. Acad. Sci. Paris Série I 331 (2000) 55-60, math. $\overline{\mathrm{S} G / 000 \overline{1}} \overline{1} \overline{0}$,

Integrable Spin Calogero-Moser Systems, math.QA/0105162i. 